\title{
Primary Exophytic Extraskeletal Osteosarcoma of the Liver: A Case Report and Literature Review
}

This article was published in the following Dove Press journal:

Risk Management and Healthcare Policy

\author{
Jing Zhang' \\ Xiuchao $\mathrm{He}^{\prime}$ \\ Wenying $\mathrm{Yu}^{2}$ \\ Fuming Ying ${ }^{3}$ \\ Jun $\mathrm{Cai}^{4}$ \\ Shengde Deng' \\ 'Department of Imaging Center, The \\ Affiliated Hospital of Medical School of \\ Ningbo University, Ningbo, Zhejiang \\ Province, 315000, People's Republic of \\ China; ${ }^{2}$ Ningbo Diagnostic Pathology \\ Center, Ningbo, Zhejiang Province, \\ 315000, People's Republic of China; \\ ${ }^{3}$ Department of Hepatobiliary Surgery, \\ The Affiliated Hospital of Medical School \\ of Ningbo University, Ningbo, Zhejiang \\ Province, 3I5000, People's Republic of \\ China; ${ }^{4}$ Department of PET-CT Diagnosis \\ Center, Mingzhou Hospital of Zhejiang \\ University, Ningbo, Zhejiang Province, \\ 315000, People's Republic of China
}

Correspondence: Shengde Deng Department of Imaging Center, The Affiliated Hospital of Medical School of Ningbo University, No. 247 of Renmin Street, Jiangbei District, Ningbo, Zhejiang Province, 315000, People's Republic of China

$\mathrm{Tel} / \mathrm{Fax}+8657455873876$

Email demgshemgde@I63.com
Introduction: Primary hepatic extraskeletal osteosarcoma (ESOS) is a rare tumor with no specific clinical manifestations, and little is known about it. Here, we describe an elderly patient with primary hepatic osteosarcoma confirmed by pathology results to raise awareness. Case Report: We report an unusual case of a 62-year-old man who presented with right upper quadrant pain. The inflammatory indicators were elevated, and alkaline phosphatase (AKP), carbohydrate antigen (CA-199 and CA-125) were slightly increased. Computed tomography images and magnetic resonance images discovered a $7.8 \times 7.4 \times 6.6 \mathrm{~cm}$ mass with irregular radiated and cotton-like tumor bone between the liver and right kidney space. Pathology revealed the mass to be primary exophytic ESOS of the liver. The patient underwent a surgical operation and standard chemotherapy and is still alive with no recurrence and metastasis to date.

Conclusion: Owing to the rarity of the tumor and the lack of clinical characteristics and specific laboratory indexes, it is difficult to make a correct diagnosis. Medical imaging features mainly behave soft tissue entity with tumor bone composition. Surgical resection combined with adjuvant chemotherapy is the main treatment for ESOS.

Keywords: extraskeletal, osteosarcoma, hepatic, tumor bone, primary

\section{Introduction}

Extraskeletal osteosarcoma (ESOS) is a relatively rare tumor with poor prognosis that has only been reported on in a few studies. It constitutes less than $4 \%$ of all types of osteosarcoma, and it can form bone-like or cartilage-like materials, which is similar to osteosarcoma histologically. ${ }^{1-3}$ The absence of a primary bone lesion is a prerequisite for the diagnosis of ESOS. ${ }^{4}$ We report the case of an elderly male patient with primary hepatic exophytic ESOS that was treated by surgical resection and adjuvant chemotherapy. He is still alive, and no recurrence and metastasis have occurred to date. Soft tissue mass with tumor bone composition on imaging presentations may help in diagnosis and further understanding in similar cases.

\section{Case Report}

A 62-year-old man presented with paroxysmal right upper quadrant pain. The pain worsened the next day and was accompanied by a body temperature of $38.2^{\circ} \mathrm{C}$. On physical examination, there was tenderness in the right upper abdomen, but no mass was palpable. The laboratory tests indicated that inflammatory indicators were elevated, AKP, CA-199 and CA-125 were slightly increased, and there were no measurable findings on the other laboratory tests. The abdominal pain eased after rehydration and anti-infection treatment. 


\section{Medical History}

The patient had had hypertension for 20 years that was managed with the help of antihypertensive drugs, but the blood pressure control was poor. Six months ago his left upper lung lobe was removed because of lung adenocarcinoma. No definite trauma history or other surgeries were detected prior to the onset of illness.

\section{Imaging}

No abnormalities were found in the abdominal imaging scan six months ago (Figure 1A). An abdominal computed tomography (CT) enhanced scan was performed for further diagnosis, and it showed that there was a $7.8 \times$ $7.4 \times 6.6 \mathrm{~cm}$ mass between the liver and right kidney space (Figure 1B-F). The tumor was a cystic, solid mass with an irregular radiated and cotton-like osteoid composition located in the eccentric side, and the osteoid appeared ill-defined and inhomogeneous. The density of the tumor increased toward its center, where the CT value was 314 $\mathrm{Hu}$. The right kidney was compressed but its cortex was not infiltrated. On enhanced magnetic resonance imaging (MRI) (Figure 2), intermediate or relatively low signals on T1WI were found, and there were no abnormal signals on DWI. The whole tumor was not enhanced in both $\mathrm{CT}$ and MR. The pararenal space was unclear with flocculent exudation, and the adjacent peritoneal adhesion was displayed. Excluding tumor bone, the rest of the tumor had a multilocular cystic structure with the septa dividing cystic spaces. No enhancement of the capsule, septa, and cystic components was discovered.

\section{Operations and Pathology}

The tumor was located in the right posterior lobe of the liver and was more exophytic in its growth pattern. Excision of the tumor, which measured approximately 70 $\times 80 \mathrm{~mm}$ including the capsule, hard texture, and adhesion to the surrounding omentum tissue, was completed by performing a partial liver lobectomy. Intraoperative ultrasonography showed no obvious space-occupying lesions in the remaining liver and no distant metastases in the abdominal and pelvic cavity. Macroscopically, the round mass was cystic and solid with a complete capsule, and the cut surface had a firm, yellow-white appearance (Figure $3 \mathrm{~A}$ and $\mathrm{B}$ ). Microscopically (Figure $3 \mathrm{C}$ and D), most tumor cells in the field of view were fusiform, diffusely arranged with common mitotic figures. The neoplasm was composed of osteoid tissues and cystic spaces and lined with a single layer of columnar epithelium or cubic epithelium. Proliferation of spindle-shaped fibroblast/myofibroblast-like cells was seen in the cyst wall, and cartilage-like components were seen locally. Pathological diagnosis was

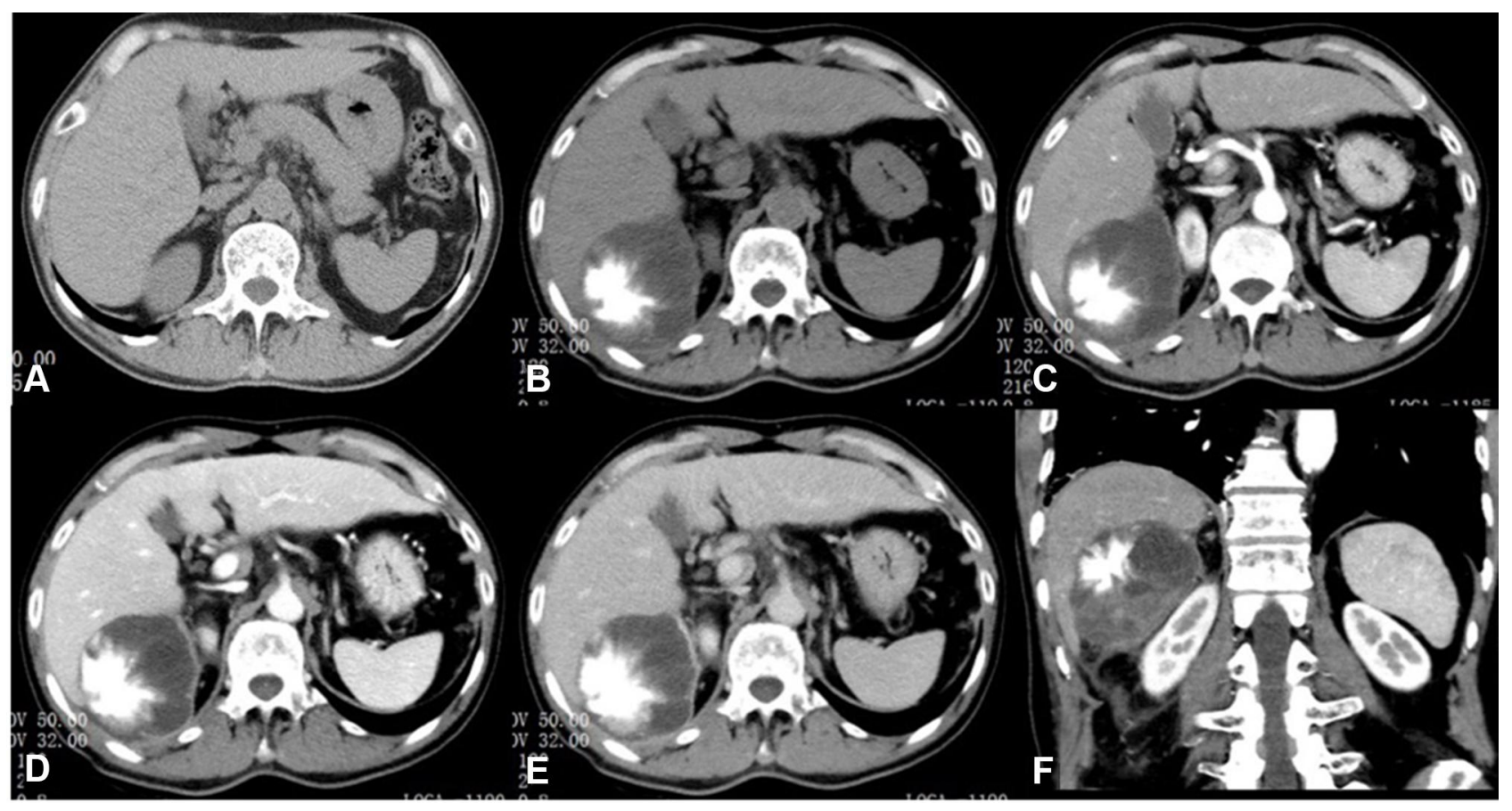

Figure I (A) Abdominal CT scan that found no abnormalities six months ago. (B-E) Abdominal enhanced CT scan (plain, arterial phase, portal phase, delayed phase in order). (F) Coronal reconstruction (arterial phase). 


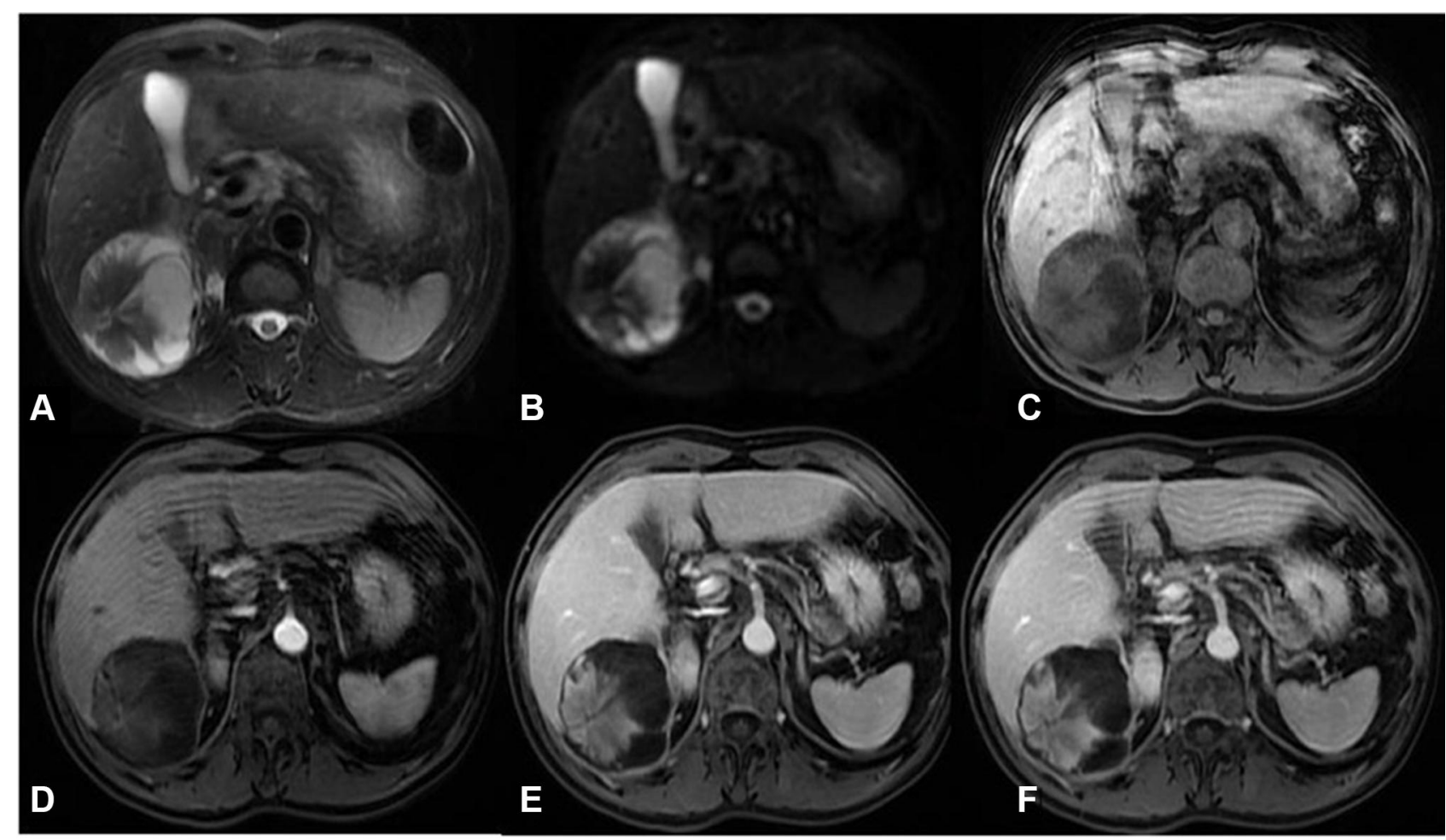

Figure 2 Enhanced MR scan of upper abdomen ((A) T2 weighted imaging (T2WI); (B) diffusion weighted imaging (DWI); (C) LAVA-flex MASK; (D) arterial phase; (E) portal phase; (F) delayed phase).

osteoblastic sarcoma; however, more tests were required to determine whether the mass was primary or metastatic. This pathology result was affirmed by experts from Fudan University Shanghai Cancer Center. The immunohistochemical analysis (Figure 4) contains the following markers: CD34, CK7, Hepatocyte, Ki-67, CK19, GPC3, P53, CK (pan), CAM5.2, MOC31, MDM2 and CEA. Among these markers, percentage of Ki-67 was 20\% and the rest were negative. Based on H\&E morphology, immunohistochemistry, and molecular detection results, the opinion at the final consultation was hepatic ESOS (osteoblastic type). The AKP, CA-199 and CA-125 had returned to normal in postoperative laboratory tests.

\section{Postoperative PET-CT}

No obvious abnormal fluorodeoxyglucose (FDG) uptake area was displayed except for the abdominal incision and tissues of the operative region (Figure 5). The patient had to undergo an adjuvant AP regimen (epirubicin $30 \mathrm{mg} / \mathrm{m}^{2} \mathrm{D} 1-$ 3 and cisplatin $150 \mathrm{mg} / \mathrm{m}^{2} \mathrm{D} 2$ ); however, the regimen was changed to albumin-bound paclitaxel $180 \mathrm{mg} / \mathrm{m}^{2}$ plus gemcitabine $1400 \mathrm{mg} / \mathrm{m}^{2}$ (D1, D8, q3W) on account of the slight return to normality of his renal function (increased urine output over 24 hours, slightly elevated creatinine, and reduced creatinine clearance rate). Currently, his condition is generally good, and he does not have any discomfort.

\section{Discussion}

ESOS is a rare malignant tumor that originates from soft mesenchymal tissue. It constitutes less than $4 \%$ of all types of osteosarcoma, and it can form bone-like or cartilagelike materials, which is similar to osteosarcoma histologically. $^{1-3}$ It occurs predominantly in elderly patients, and the mean age has been reported as 54 years. ${ }^{5}$ Though ESOS most commonly affects legs and hips, there are case reports of it occurring in the thyroid, kidney, gallbladder, bladder, breast, uterus, and lung. ${ }^{2,6-11}$ However, it is not clear where osteosarcoma stem cell comes from either in bone or in extraskeletal cases and the pathogenesis is still under research. There are studies indicating that trauma and exposure to radiation may be the main risk factors for ESOS. In addition, trauma and exposure to radiation may be the main risk factors for ESOS. Our patient has no explicit trauma history but has a history of lung cancer, which was removed under Videoassisted Thoracoscopic Surgery (VATS) six months ago without postoperative radiation therapy. Moreover, no other suspect lesions were indicated in the PET-CT test 


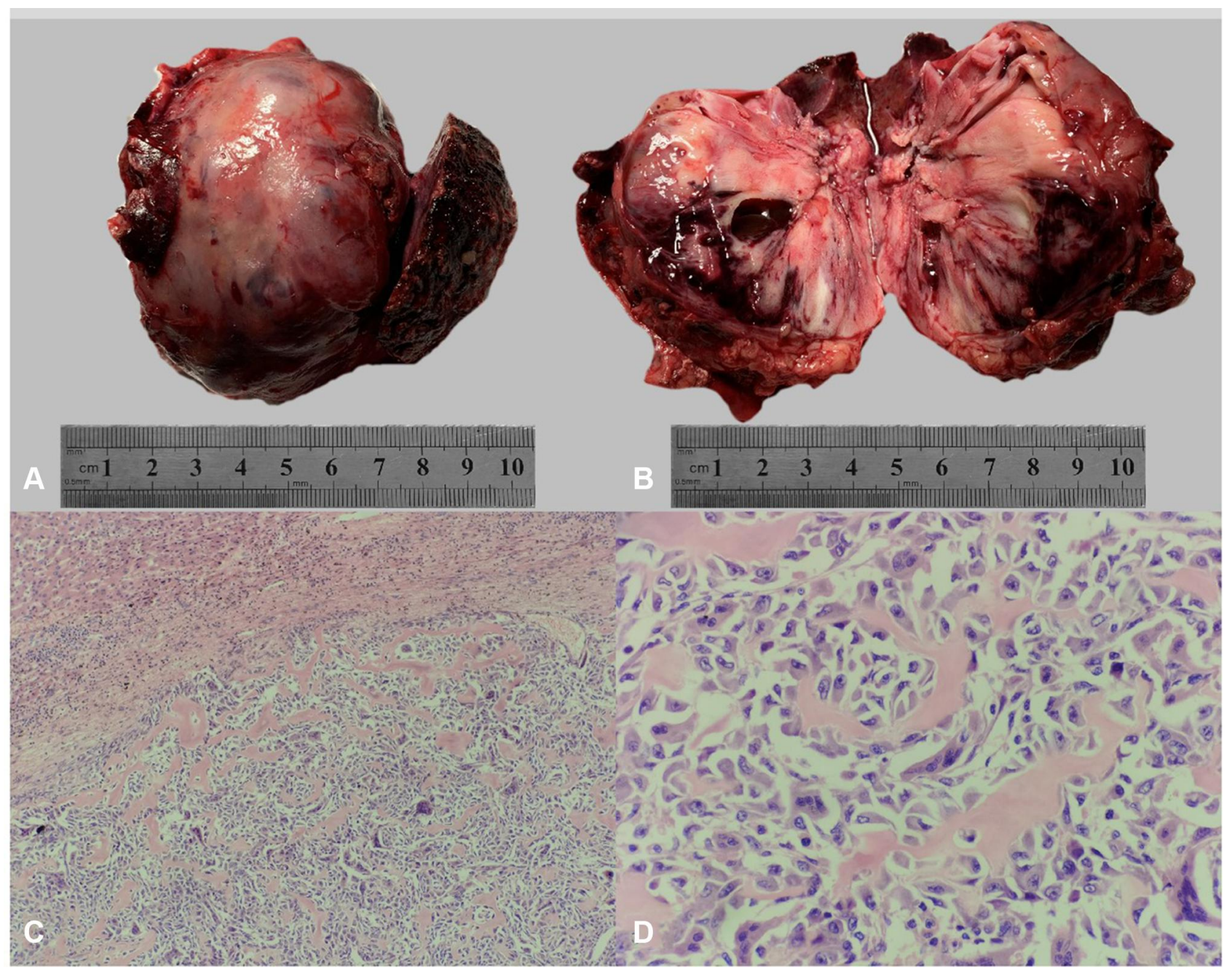

Figure 3 (A) Resection of the spherical tumor and part of the liver. (B) Cut face of the resection specimen that shows a firm, yellow-white appearance. (C) Low magnification: liver tissues (above), fibrous capsule (middle), tumor tissues (below). (D) High magnification: neoplastic cells with marked pleomorphism and lace-like or trabecular osteoid tissues.
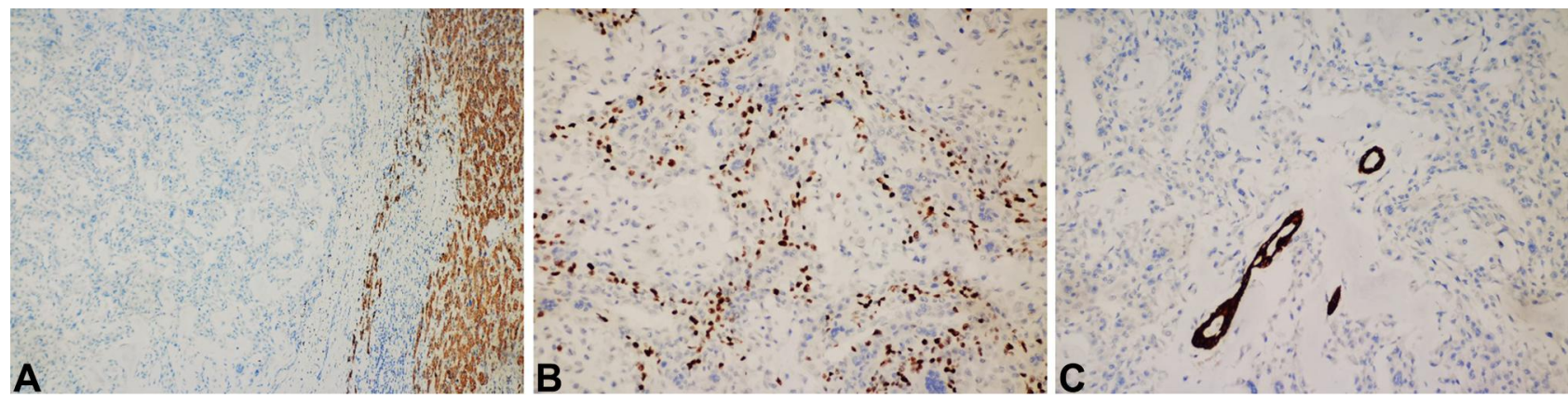

Figure 4 The immunohistochemical analysis ((A) low-power immunohistochemical, hepatocyte, tumor negative on the left, normal liver positive on the right; (B) medium immunohistochemistry, Ki-67; (C) medium-power immunohistochemistry, CK7, residual bile ducts positive, tumor negative).

within 20 days after surgery, which suggests that the tumor arises from the liver and is exophytic in its growth pattern, which has not been reported yet at home and abroad. Tamang et $\mathrm{al}^{12}$ found five cases of primary hepatic osteosarcoma patients who had underlying hepatic cirrhosis. Sumiyoshi and Niho ${ }^{13}$ suggested that tumors may occur in proliferative mesenchymal tissues in cirrhosis, and therefore they conjectured that cirrhosis might be 


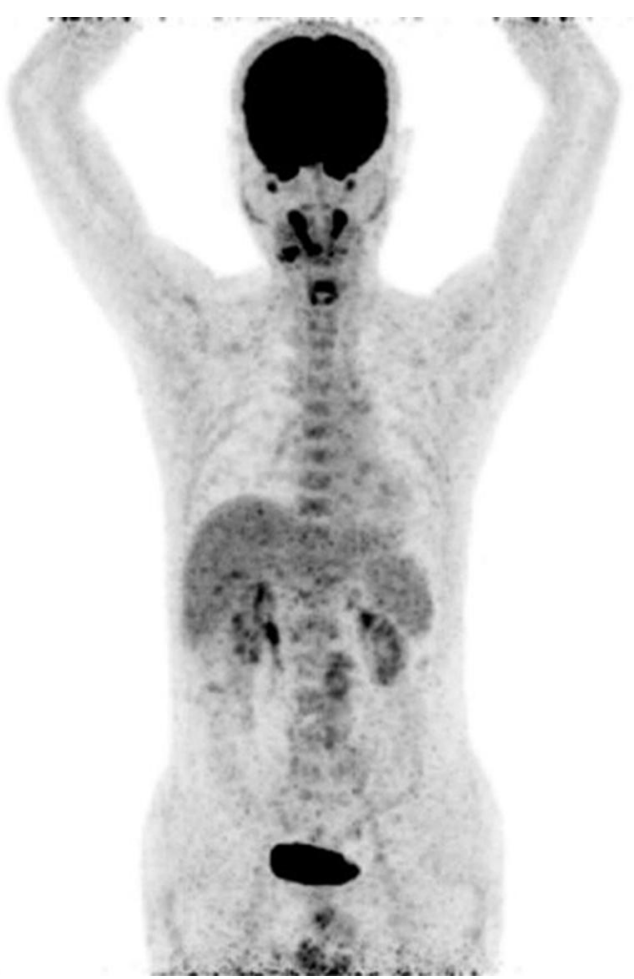

Figure 5 No obvious abnormal FDG uptake area was displayed except for the abdominal incision and tissues of the operative region.

a risk factor of this rare tumor. Although our patient did not show significant characteristics of liver cirrhosis on imaging examination, the liver was found to be fibrotic, firm, and irregular in outline during the operation. Moreover, the $\mathrm{HBsAg}, \mathrm{HBeAb}$ and $\mathrm{HBcAb}$ were positive; therefore, we speculated that liver cirrhosis might be at an early stage, which was in accordance with the conclusion of Tamang et al. ${ }^{12}$ Primary hepatic ESOS is occult in onset with atypical manifestations, such as a gradually increasing abdominal mass, which may be associated with pain. The prognosis is poor because of the short course, rapid progress, and high likelihood of distant metastasis. ${ }^{14}$ It is worth noting that in this case, no abnormalities were found in the abdominal imaging scan six months ago, which is a measure of poor prognosis.

CT and MR are the main important imaging tools of ESOS. The CT manifestations were extraskeletal mass with varying degrees of tumor bones (detection rate of $68 \%$ ), which is a prominent characteristic of ESOS and has a certain prompt effect for diagnosis. The tumor bone has morphological diversity that can be described as cotton-like, ivory-like, spicules or hybrid type. CT can sensitively distinguish the peripheral part of weak osteogenesis, which is superior to X-ray plain film and MR. Most of the tumor bone shows an iso- or hypo- intense signal on T1 weighted imaging (T1WI), and the central part is usually lower than the peripheral part, which represents abundant tumor cells. The tumor may undergo necrosis, cyst degeneration, and bleeding and may be accompanied by airliquid level when it has an infection, which makes the tumor's density/signal mixed. On the enhanced scan, the solid component of the mass showed evident enhancement generally without visible clearance; some tumors may also manifest mild or no enhancement. ${ }^{15}$ The signal changes displayed on MR are able to reflect the general pathological changes of ESOS; however, it is not as sensitive as CT to the display of micro tumor bone and calcification. In our case, the tumor performed multilocular cystic-solid configuration, with exudation in the right pararenal space presented on imaging. Furthermore, our patient had fever at the time of onset, and laboratory tests indicated an increase in inflammation indicators; therefore, we considered it as an infected tumor. ESOS is prone to surrounding invasion or distant metastasis, and pulmonary metastasis is the most common (37 80\%). A CT scan is helpful for the detection of lung metastasis. Metastasis in the lungs and other parts of our patient has not yet been found. The prognosis of ESOS is poor. ${ }^{16,17}$ Surgical resection is the main method of treatment, and postoperative radiotherapy is combined with chemotherapy to prolong survival; ${ }^{18,19}$ however, the overall effect is not good. Lee et $\mathrm{al}^{20}$ reported that the 5 -year survival rate for a group of patients diagnosed with ESOS was only $37 \%$, and most patients died within 2 to 3 years after the first diagnosis. Currently, there is no paper about the prognosis of primary hepatic ESOS at home or abroad.

\section{Conclusion}

In the existing literature, there are few case reports on primary ESOS, and there are even fewer reports concerning primary hepatic ESOS, particularly exophytic ESOS, for which there is a lack of known etiology and typical clinical manifestations. Diagnosis of ESOS still relies on pathology and immunohistochemistry. Hepatic ESOS should be differentiated from hepatic metastasis of osteosarcoma, malignant fibrous histiocytoma, chondrosarcoma, and rhabdomyosarcoma. ${ }^{21}$ It should be considered when an extraskeletal soft tissue mass with osteoblastic components or tumor bone is found on imaging.

\section{Ethics Approval and Consent to Participate}

This study was conducted with approval from the Ethics Committee of The Affiliated Hospital of Medical School 
of Ningbo University to publish the case details. This study was conducted in accordance with the declaration of Helsinki. Written informed consent was obtained from the patient to have the case details and any accompanying images published.

\section{Disclosure}

The authors report no conflicts of interest in this work.

\section{References}

1. Yugawa K, Yoshizumi T, Mano Y, et al. Primary intrahepatic cholangiocarcinoma with sarcomatous stroma: case report and review of the literature. Surg Case Rep. 2018;4(1):138. doi:10.1186/s40792-0180543-z

2. Bane BL, Evans HL, Ro JY, et al. Extraskeletal osteosarcoma. A clinicopathologic review of 26 cases. Cancer. 1990;65 (12):2762-2770. doi:10.1002/1097-0142(19900615)65:12<2762:: AID-CNCR2820651226>3.0.CO;2-K

3. Siddappa JK, Singla S, Jain A, Kumar A, Rare A. Case of primary osteosarcoma of urinary bladder. J Clin Imaging Sci. 2012;2:82. doi:10.4103/2156-7514.105145

4. Johnson C, Kim Y. Hepatic extraskeletal osteosarcoma with systemic metastasis in a dog. Korean $J$ Vet Res. 2013;53(1):61-64. doi:10.14405/kjvr.2013.53.1.061

5. Choi LE, Healey JH, Kuk D, Brennan MF. Analysis of outcomes in extraskeletal osteosarcoma: a review of fifty-three cases. $J$ Bone Joint Surg Am. 2014;96(1):e2. doi:10.2106/JBJS.M.00339

6. Nawabi A, Rath S, Nissen N, et al. Primary hepatic osteosarcoma. $J$ Gastrointest Surg. 2009;13(8):1550-1553. doi:10.1007/s11605-009$0852-4$

7. Huang C, Zhu X, Xiong W, Zhao X, Xu R. A case report of primary osteosarcoma originating from kidney. Medicine (Baltimore). 2019;98 (4):e14234. doi:10.1097/MD.0000000000014234

8. Zembala-Nożyńska E, Lange D. Primary osteosarcoma of the thyroid gland - a case report. Contemp Oncol (Pozn). 2013;17(1):97-99. doi:10.5114/wo.2013.33783

9. Tsukasaki N, Mori T, Yasukawa S, Konishi E, Kokabu T, Kitawaki J. Primary osteosarcoma of the uterine corpus: a case report. J Obstet Gynaecol Res. 2016;42(11):1604-1608. doi:10.1111/jog.13079
10. Dekkers IA, Cleven A, Lamb HJ, Kroon HM. Primary osteosarcoma of the breast. Radiographics. 2019;39(3):626-629. doi:10.1148/ rg.2019180181

11. Mremi A, Pyuza JJ, Bartholomeo N, Andongolile AA, Yahaya JJ. Diagnosis of primary osteosarcoma of the urinary bladder in a resource-limited setting: a case report and review of the literature. J Surg Case Rep. 2020;2020(7):rjaa236. doi:10.1093/jscr/rjaa236

12. Tamang TG, Shuster M, Chandra AB. Primary hepatic osteosarcoma: a rare cause of primary liver tumor. Clin Med Insights Case Rep. 2016;9:31-33. doi:10.4137/CCRep.S38384

13. Sumiyoshi A, Niho Y. Primary osteogenic sarcoma of the liver-report of an autopsy case. Acta Pathol Jpn. 1971;21(2):305-312. doi:10.1111/j.1440-1827.1971.tb00126.x

14. Usui G, Hashimoto H, Kusakabe M, et al. Intrahepatic carcinosarcoma with cholangiocarcinoma elements and prominent bile duct spread. Int J Surg Pathol. 2019;27(8):900-906. doi:10.1177/ 1066896919855766

15. Hoch M, Ali S, Agrawal S, Wang C, Khurana JS. Extraskeletal osteosarcoma: a case report and review of the literature. J Radiol Case Rep. 2013;7(7):15-23. doi:10.3941/jrcr.v7i7.1245

16. Berner K, Bjerkehagen B, Bruland ØS, Berner A. Extraskeletal osteosarcoma in Norway, between 1975 and 2009, and a brief review of the literature. Anticancer Res. 2015;35(4):2129-2140.

17. Nystrom LM, Reimer NB, Reith JD, Scarborough MT, Gibbs CP Jr. The treatment and outcomes of extraskeletal osteosarcoma: institutional experience and review of the literature. Iowa Orthop J. 2016;36:98-103.

18. Kurita D, Mokuno Y, Matsubara H, et al. Primary hepatic carcinosarcoma with multimodal treatment. Nagoya J Med Sci. 2018;80 (3):423-429. doi:10.18999/nagjms.80.3.423

19. Park SH, Choi SB, Kim WB, Song TJ. Huge primary osteosarcoma of the liver presenting an aggressive recurrent pattern following surgical resection. J Dig Dis. 2009;10(3):231-235. doi:10.1111/ j.1751-2980.2009.00391.x

20. Lee JS, Fetsch JF, Wasdhal DA, Lee BP, Pritchard DJ, Nascimento AG. A review of 40 patients with extraskeletal osteosarcoma. Cancer. 1995;76(11):2253-2259. doi:10.1002/10970142(19951201)76:11<2253::AID-CNCR2820761112>3.0.CO;2-8

21. Mc Auley G, Jagannathan J, O'Regan K, et al. Extraskeletal osteosarcoma: spectrum of imaging findings. AJR Am J Roentgenol. 2012;198(1):W31-7. doi:10.2214/AJR.11.6927
Risk Management and Healthcare Policy

\section{Publish your work in this journal}

Risk Management and Healthcare Policy is an international, peerreviewed, open access journal focusing on all aspects of public health, policy, and preventative measures to promote good health and improve morbidity and mortality in the population. The journal welcomes submitted papers covering original research, basic science, clinical \& epidemiological studies, reviews and evaluations, guidelines, expert opinion and commentary, case reports and extended reports. The manuscript management system is completely online and includes a very quick and fair peer-review system, which is all easy to use. Visit http://www.dovepress.com/testimonials.php to read real quotes from published authors. 